\title{
Transaortic approach for combined aortotricuspid valve endocarditis
}

\author{
Jama Jahanyar, MD, PhD, Gaby Aphram, MD, Laurent de Kerchove, MD, PhD, and \\ Gebrine El Khoury, MD, PhD, Brussels, Belgium
}

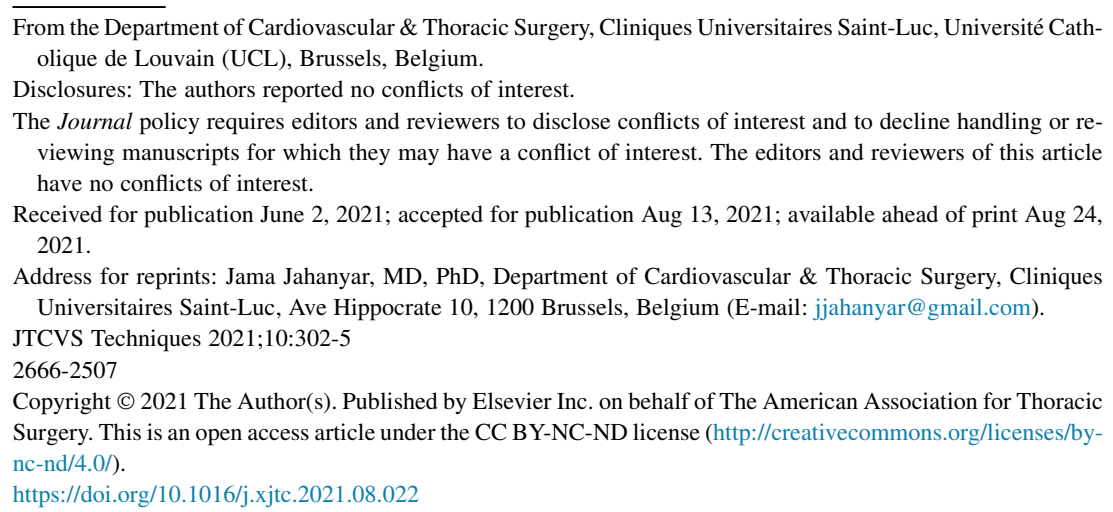

V Video clip is available online.

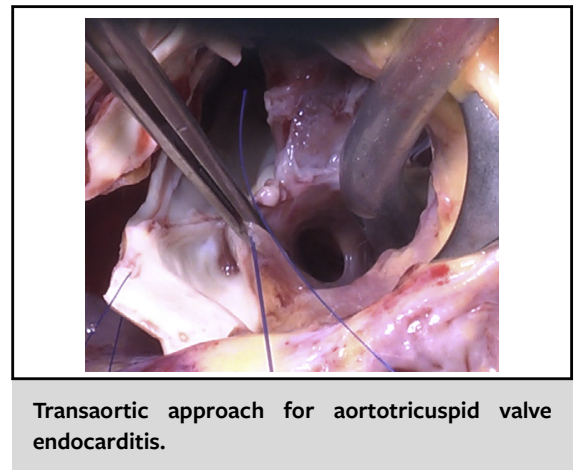

CENTRAL MESSAGE

Repair of the tricuspid valve in combined aortotricuspid valve endocarditis is feasible through the aortic root/left ventricular outflow tract.

See Commentaries on pages 306 and 307.
Aortotricuspid valve infective endocarditis (IE) is rare, and surgical reports are limited to a few. The traditional approach is a combined transaortic and transatrial valve repair or replacement.

In a recent study, 1-year mortality rates in native multivalve endocarditis were close to $30 \%$, and there has been a recent shift in pathogens toward Staphylococcus. ${ }^{1}$ Independent predictors of mortality were older age, Staphylococcus aureus, heart failure, septic shock, and persistent bacteremia. Herein, we report the management of a young patient with combined aortotricuspid valve endocarditis and our nontraditional, albeit successful, surgical approach.

\section{CLINICAL SCENARIO}

A 30-year-old male patient presented to the hospital with left-sided hemiplegia and a few days' history of persistent fevers. On physical examination, he was noted to have painful lesions on his fingers (Osler nodes). He had no significant medical or surgical history. His blood cultures grew $S$ aureus, and he was noted to be thrombocytopenic. Computed tomography scans identified right-hemispheric septic brain emboli, and multiple splenic and renal emboli.

A transthoracic echocardiogram was consistent with a combined aortotricuspid valve endocarditis, in the setting of a bicuspid aortic valve (BAV), with right/left-coronary cusp fusion, and moderate aortic valve regurgitation. The aortic valve vegetation measured $38 \times 13 \mathrm{~mm}$ in size, and the tricuspid valve vegetation was $22 \times 13 \mathrm{~mm}$. The patient was therefore taken to the operating room to undergo a combined aortic and tricuspid valve repair, possible replacement.

\section{SURGICAL TECHNIQUE}

The patient was placed on cardiopulmonary bypass, via standard central aortic and bicaval cannulation. An aortic root vent was placed to deliver antegrade cardioplegia. The heart was then crossclamped and arrested with normothermic blood cardioplegia, and a left ventricular vent was placed via the right upper pulmonary vein.

An aortotomy was performed. The aortic valve was inspected and large vegetations were noted (Figure 1, $A$ and $B)$. The vegetations were mainly located on the right coronary cusp and were also adjacent to the membranous ventricular septum. The right and left coronary artery were mobilized, and an ectopic origin of the right was noted, originating just above the raphe.

The membranous septum was then examined and found to be phlegmonous with an annular abscess (Figure 1,C). This was therefore extensively debrided 

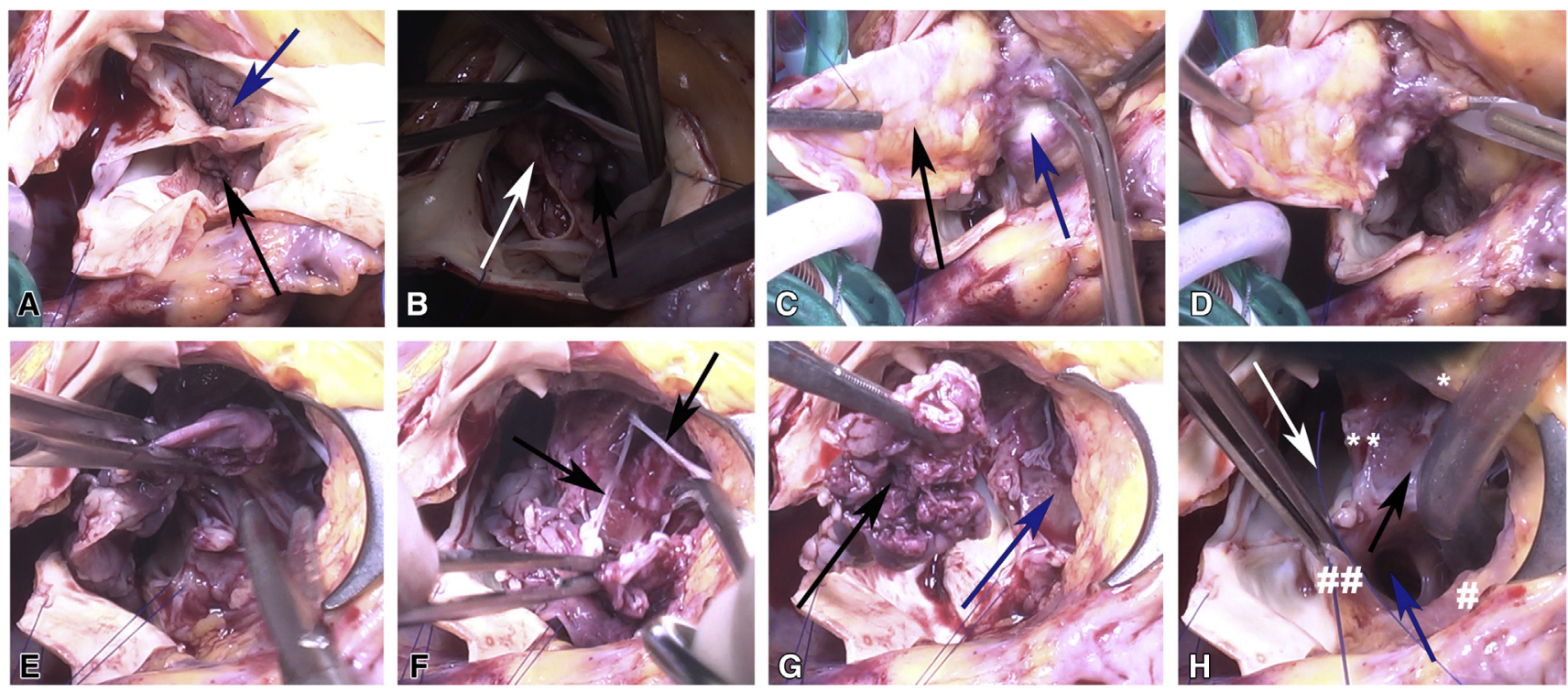

FIGURE 1. Resection of infected tissues in combined aortotricuspid valve endocarditis. A, Bicuspid aortic valve with right/left coronary cusp fusion. Blue arrow, vegetations above the right portion of the fused cusp. Black arrow, vegetations below the fused cusp. B, Large vegetations below the fused cusp. Black arrow, vegetations below the right portion of the fused cusp. White arrow, vegetations below the left portion of the fused cusp. C, Annular abscess. Black arrow, right/non commissure. Blue arrow, abscess in membranous septum. D, Debridement of membranous septum. E, Debridement of vegetations on tricuspid valve septal leaflet. F, Arrows depict anterior tricuspid leaflet chords. G, Black arrow, specimen of tricuspid valve vegetations, necrotic myocardium, parts of anterior and septal leaflet. Blue arrow, remnant septal leaflet. $\mathrm{H}$, Completely debrided surgical situs. White arrow, left ventricular outflow tract. Black arrow, septal leaflet. Blue arrow, coronary sinus. *Right ventricular free wall; **interventricular septum edge; \#right atrium free wall; \#\#atrial septum edge.

(Figure $1, D$ ). With this, the right atrium and ventricle were entered and the tricuspid valve exposed (Figure 1, $E)$. There were extensive vegetations on the septal and anterior leaflet of the tricuspid valve. The vegetations were resected sharply, attempting to preserve as much leaflet tissue and subvalvular apparatus as possible (Figure 1, F-H).

The tricuspid valve was then repaired by identifying the free margins of the septal and anterior leaflets (Video 1). The valve was essentially bicuspidized by sewing the free margins together for a few millimeters toward the anteroseptal commissure and closing the remaining triangular

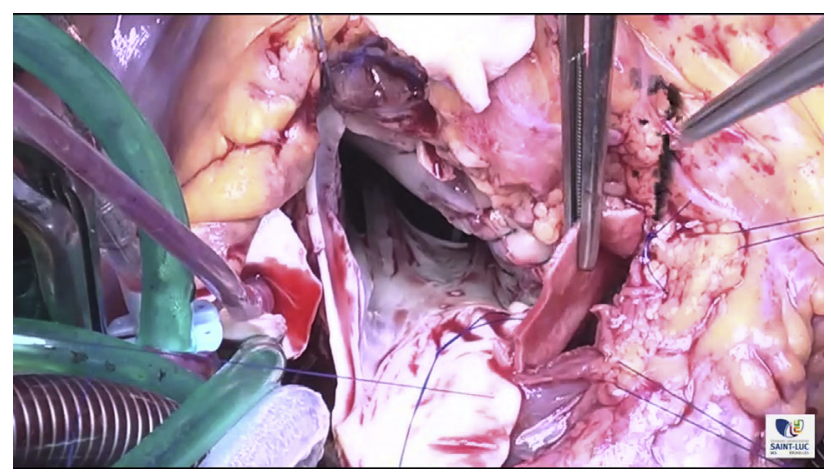

VIDEO 1. Transaortic approach for combined aortotricuspid valve endocarditis: tricuspid valve repair and aortic valve replacement with homograft. Video available at: https://www.jtcvs.org/article/S2666-2507 (21)00579-4/fulltext. defect with a bovine pericardial patch, together with closure of the membranous ventricular septal defect (Figure 2, $A-D)$.

Hence, a bovine pericardial patch was then sewn to the interventricular septum, beginning at the level of the septal tricuspid annulus. It was taken around the right ventricular free wall, and this suture line also included the anterior tricuspid valve leaflet edge. The next suture line then, sewed the patch to the septal annulus including the free edge of the septal leaflet. With this, the ventricular septal defect and tricuspid valve repair was completed. This was followed by sewing of the patch to the atrial septum and right atrial free wall, to close the atrial defect.

A homograft was sewn to the aortic annulus with a 4.0 PROLENE suture in a running fashion and the coronaries were reimplanted (Figure 2, $E-H$ ). The distal aortic anastomosis was completed and the patient was weaned off cardiopulmonary bypass.

Written informed consent for publication was obtained from the patient. No institutional review board approval is required by our institution for case reports.

\section{COMMENTS}

This is the first report of this surgical approach for aortotricuspid valve endocarditis. In this patient, the infection extended into the aortic annulus, leading to an abscess of the membranous septum, subsequently extending into the 

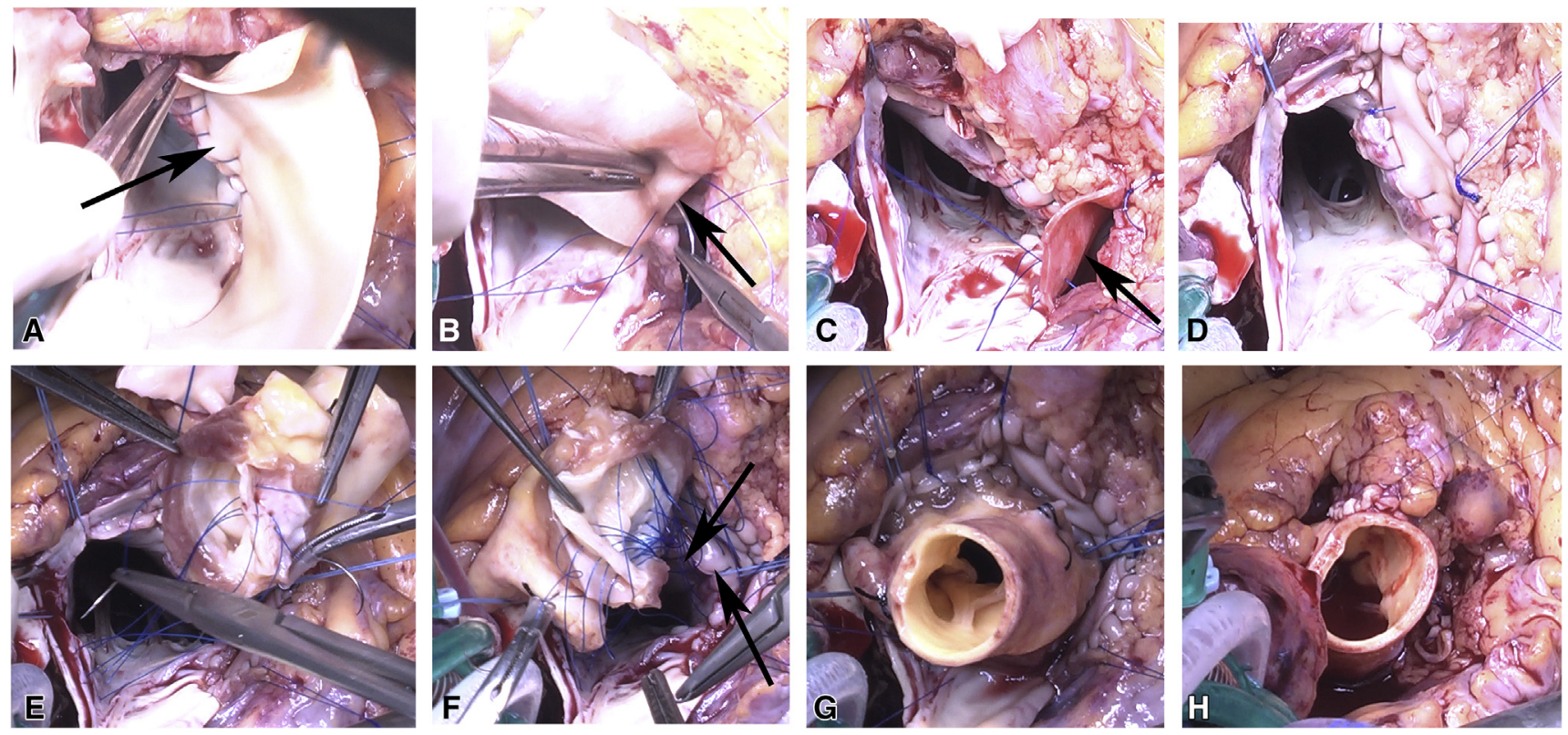

FIGURE 2. Surgical repair. A, Patch repair of VSD. Black arrows suture line on the interventricular septum. B, Completing repair of VSD and tricuspid valve. Black arrows, suture line on septal tricuspid valve annulus, incorporating free edge of septal leaflet. C, Completing closure of atrial defect. Black arrows, suture line on right atrial free edge. D, Completed repair of VSD, tricuspid valve, and right atrium. E, Placement of aortic homograft. F, Black arrows, suture line of the homograft on the bovine pericardial patch. G, Fully seated homograft. H, Homograft after left and right coronary artery reimplantation.

right heart, and affecting the anterior and septal leaflets of the tricuspid valve.

In our experience, whenever we find an abscess of the tricuspid valve annulus, it is almost exclusively in the fibrous membranous septum. Moreover, in the mitral valve it's also almost exclusively in the aortomitral curtain and hence the fibrous portion of the mitral annulus. The muscular portions of the tricuspid and mitral valve annulus are mostly spared. However, in the setting of mitral annular calcifications, we do observe abscess formation of the posterior mitral annulus. Tricuspid annular calcifications almost never occur, and accordingly we do not see abscesses in the muscular tricuspid annulus. And when an abscess of the fibrous portion of the atrioventricular valves occurs, they are almost always located on the ventricular side of the valves, and accordingly are easier to treat from the aortic side.

Thus, due to these intraoperative findings, and the need for debridement of the membranous septum, the surgical approach shifted from a combined transaortic- and transatrial-, to the transaortic-approach only.

We believe that a major pitfall in endocarditis surgery is not to grasp the entire extent of the infection correctly, and to thus not adequately resect all infected/necrotic tissues to achieve healthy margins. Therefore, the infection may be ongoing after surgery, and more importantly, the repairs are then performed in unhealthy tissues, which can lead to breaking down of the repairs later on (eg, valve prosthesis dehiscence). We therefore follow an aggressive approach, with resection of all necrotic tissues, followed by surgical repair, which is tailored to the specific circumstances. In this patient, an additional transatrial approach was unnecessary, as everything was repairable through the aortic valve. However, if the tricuspid valve would have been regurgitant after the repair was completed, or if there were any intraoperative concerns regarding the function of the valve, then a subsequent transatrial repair would have been undertaken.

We don't advocate for using this approach in every case of aortotricuspid valve endocarditis. Yet, when the aortic annulus is involved, which is often the case in $\mathrm{BAV},{ }^{2}$ then extensive debridement is sometimes necessary to resect all infected tissues, so a durable repair can be achieved. In our experience, we have performed this approach in roughly a handful of patients over the last 3 decades, with varying degrees of interventricular septal and tricuspid valve involvement. Preoperatively, there was no clear indication that the membranous septum was affected. However, whenever there is combined aortotricuspid valve endocarditis, and the affected tricuspid leaflet is in direct continuity with the membranous septum, then an abscess can be expected.

Interestingly, BAV is associated with IE in younger patients, with fewer comorbidities, a greater rate of Staphylococcal infections, and perivalvular complications such as periannular abscess. ${ }^{3}$ And since BAV is the most common congenital valvular heart disease among adults, with a prevalence of $0.5 \%$ to $2 \%$, this patient population constitutes a significant proportion of patients with IE, who are more 
likely than tricuspid aortic valves to have an aortic root abscess, and to require valve replacements. ${ }^{2}$ In our experience in BAV the most commonly affected cusp is the noncoronary. We often find perforations of this cusp, which would then require a patch repair. However, in most BAVs the noncoronary cusp is the healthy unfused cusp, and the fused cusp is often foreshortened with a raphe and is hence abnormal. We therefore rarely repair BAVs in endocarditis, since it would leave the patient with two abnormal cusps with questionable long-term durability.

Many cardiac surgeons are reluctant to operate in active endocarditis, due concerns of fragile tissues. We, however, believe that fragile tissues are just a myth, and that these tissues are necrotic and need to be removed for a durable repair. Surgeons must be knowledgeable regarding the anatomy of the aortic root, and we believe that this description of the transaortic approach furthers the surgeon's anatomical understanding of the aortic root behind the right/non commissure. "Judgment evolves from knowledge" ; hence, surgeons who are comfortable with the anatomy are more inclined to perform a durable repair, instead of a limited resection with remaining infected/necrotic tissues.
Although this condition is associated with a high mortality, this patient is doing well 5 years after his surgery, with complete resolution of his neurologic symptoms. We believe that his young age was certainly protective; however, the key was the curative surgical resection of all infected tissues. IE is a challenging condition, with high morbidity and mortality, as well as surgical complexity. Herein, we have presented an alternative transaortic approach for a multivalve pathology, with an excellent outcome.

\section{References}

1. Cresti A, Chiavarelli M, Scalese M, Nencioni C, Valentini S, Guerrini F, et al Epidemiological and mortality trends in infective endocarditis, a 17-year population-based prospective study. Cardiovasc Diagn Ther. 2017;7: 27-35.

2. Kiyota Y, Della Corte A, Montiero Vieira V, Habchi K, Huang CC, Della Ratta EE, et al. Risk and outcomes of aortic valve endocarditis among patients with bicuspid and tricuspid aortic valves. Open Heart. 2017;4:e000545.

3. Becerra-Munoz VM, Ruiz-Morales J, Rodriguez-Bailon I, Sánchez-Espín G, López-Garrido MA, Robledo-Carmona J, et al. Infective endocarditis in patients with bicuspid aortic valve: clinical characteristics, complications, and prognosis. Enferm Infecc Microbiol Clin. 2017;35:645-50.

4. David TE. For everything there is a season. J Thorac Cardiovasc Surg. 2005;130: 961-5. 